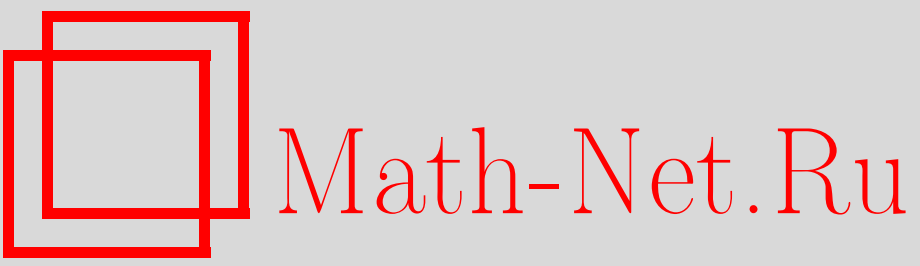

М. О. Катанаев, Топологически-нетривиальные одномерные решения в модели Скирма, ТМФ, 2004, том 138, номер 2, 193-208

DOI: https://doi.org/10.4213/tmf22

Использование Общероссийского математического портала Math-Net.Ru подразумевает, что вы прочитали и согласны с пользовательским соглашением

http://www . mathnet.ru/rus/agreement

Параметры загрузки:

IP : 52.6 .47 .48

26 апреля 2023 г., 08:08:05 
ТЕОРЕТИЧЕСКАЯ

И МАТЕМАТИЧЕСКАЯ

ФИЗИКА

Том 138, № 2

февраль, 2004

(C) 2004 г.

М.О. Катанаев*

\section{ТОПОЛОГИЧЕСКИ-НЕТРИВИАЛЬНЫЕ ОДНОМЕРНЫЕ РЕШЕНИЯ В МОДЕЛИ СКИРМА}

Модель Скирма рассматривается с использованием явной параметризации группы вращений $\mathbb{S O}(3)$ элементами ее алгебры. Поскольку фундаментальная группа группы $\mathbb{S O}(3)$ равна $\mathbb{Z}_{2}$, то топологически-нетривиальные решения возникают уже в одномерном случае. Явно найдены и проанализированы статические одномерные решения. Среди них есть топологически-нетривиальные решения с конечной энергией. Предложен новый класс проективных моделей, пространством-мишенью которых являются произвольные действительные проективные пространства $\mathbb{R P}^{d}$.

Ключевые слова: топологические солитоны, модель Скирма.

\section{1. ВВЕДЕНИЕ}

Модель Скирма [1] является одной из фундаментальных моделей современной математической физики, которая находит применение в разных областях физики от теории элементарных частиц до физики твердого тела. Первоначально модель была предложена в терминах четырех скалярных полей, принимающих значения на трехмерной сфеpe $\mathbb{S}^{3}$. Поскольку как многообразие сфера $\mathbb{S}^{3}$ диффеоморфна группе $\mathbb{S} \mathbb{U}(2)$, то лагранжиан модели был также записан в терминах элементов алгебры $\mathfrak{s}(2)$. Так как фундаментальная и вторая гомотопическая группы унитарных групп $\mathbb{S} \mathbb{U}(n), n \geqslant 2$, и сфер $\mathbb{S}^{n}$, $n \geqslant 3$, тривиальны, то в соответствующих моделях не может существовать одномерных и двумерных топологически-нетривиальных решений. Топологически-нетривиальные решения при такой параметризации модели Скирма связаны с третьей гомотопической группой $\pi_{3}\left(\mathbb{S}^{3}\right)=\mathbb{Z}$. Однако в явном виде эти решения не найдены.

В последуюшие годы большое внимание привлекли также $\mathbb{S} \mathfrak{O}(n)$-модели, в которых пространством-мишенью являются сферы произвольной размерности $\mathbb{S}^{n-1}$, для которых группа вращений $\mathbb{S O}(n)$ есть группа симметрии. Обзор этих моделей и библиографию можно найти в монографиях [2]. В дальнейшем мы будем называть эти модели $\mathbb{S}^{n}$-моделями, зарезервировав термин $\mathbb{S} \mathfrak{O}(n)$-модели для того случая, когда пространством-мишенью является само групповое многообразие.

* Математический институт им. В. А. Стеклова РАН, Москва, Россия. E-mail: katanaev@mi.ras.ru 
Топологические солитоны низшей размерности существуют для $\mathbb{S}^{1}$-моделей, для которых $\pi\left(\mathbb{S}^{1}\right)=\mathbb{Z}$. Это, например, хорошо известные кинки в модели синус-Гордон. Топологически-нетривиальные решения для более высоких гомотопических групп появляются в $\mathbb{S}^{2}$-модели. Для этих решений вторая гомотопическая группа нетривиальна: $\pi_{2}\left(\mathbb{S}^{2}\right)=\mathbb{Z}$. Соответствуюшие статические решения с нетривиальным топологическим зарядом были найдены и проанализированы в [3].

Как уже было отмечено, лагранжиан модели Скирма может быть записан в терминах элементов алгебры $\mathfrak{s u}(2)$. Так как алгебры групп $\mathbb{S} U(2)$ и $\mathbb{S} O(3)$ изоморфны, то модель Скирма можно рассматривать как $\mathbb{S O}(3)$-модель, пространством-мишенью которой является само групповое многообразие. С этой целью в настоящей статье используется известная (см., например, [4]), но не очень широко применяемая явная параметризация группы врашений $\mathbb{S O}(3)$, т.е. мы работаем непосредственно с трехмерным групповым многообразием. Поскольку фундаментальная группа группы врашений $\mathbb{S} \mathfrak{O}(3)$ нетривиальна $\left(\pi(\mathbb{S} \mathbb{O}(3))=\mathbb{Z}_{2}\right)$, то топологически-нетривиальные решения появляются уже в одномерном случае. Соответствуюшие статические решения найдены в явном виде. Проведен их анализ и сравнение со статическими решениями в $\mathbb{S}^{2}$-модели.

Явная параметризация группы $\mathbb{S} \mathbb{O}(3)$, используемая в настояшей статье, позволяет провести обобшение. Хорошо известно, что групповое многообразие $\mathbb{S O}(3)$ диффеоморфно трехмерному проективному пространству $\mathbb{R}^{3}$ (см., например, [5]). Это позволяет обобшить $\mathbb{S} \mathbb{O}(3)$-модель Скирма на случай произвольных проективных пространств над полем вешественных чисел, т.е. когда пространством-мишенюю является произвольное проективное пространство $\mathbb{R P}^{n}=\mathbb{S}^{n} / \mathbb{Z}_{2}$. Его можно параметризовать точками евклидова пространства $\left\{\omega^{i}\right\} \in \mathbb{R}^{n}, i=1, \ldots, n$, лежашими внутри шара $\omega=$ $\sqrt{\omega^{i} \omega_{i}} \leqslant \pi$, у которого отождествлены диаметрально противоположные точки граничной сферы радиуса $\pi$. При такой параметризации проективных пространств лагранжиан $\mathbb{R P}^{n}$-моделей зависит от $n$ полей $\omega^{i}(x)$, и инвариантен относительно дискретных локальных преобразований $\omega^{i} \rightarrow \omega^{i}+2 \pi \omega^{i} / \omega$. В отличие от калибровочных моделей, в каждой точке пространства-времени группой симметрии является не группа Ли, а дискретная группа $\mathbb{Z}$ сдвигов на постоянный вектор. При этом представление группы сдвигов локально и зависит от точки пространства-времени, так как направление вектора меняется непрерьвно.

Мы начнем рассмотрение с описания явной параметризации группы $\mathbb{O}(3)$. В разделах 3,4 и 5 мы рассмотрим лагранжианы соответственно для $\mathbb{S} \mathbb{O}(3)$-, $\mathbb{S} \mathbb{U}(2)-$ и $\mathbb{S}^{2}$-моделей и обсудим их связь. В разделах 6 и 7 будут найдены статические решения для $\mathbb{S}^{2}$ - и $\mathbb{S} \mathbb{O}(3)$-моделей и проведено их сравнение. В восьмом разделе $\mathbb{S O}(3)$-модель будет обобщена на случай произвольных действительных проективных пространств $\mathbb{R}^{d}$.

\section{2. ПАРАМЕТРИЗАЦИЯ ГРУППЫ ТРЕХМЕРНЫХ ВРАШЕНИЙ}

При проведении расчетов с группой $\mathbb{O}(3)$ удобно использовать явную параметризацию элементов группы элементами ее алгебры. Элемент алгебры $\mathfrak{s o}(3)$ может быть представлен в виде произвольной антисимметричной $(3 \times 3)$-матрицы

$$
(\omega \varepsilon)_{i}{ }^{j}=\left(\omega^{k} \varepsilon_{k}\right)_{i}{ }^{j}=\omega^{k} \varepsilon_{k i}{ }^{j} \in \mathfrak{s o}(3),
$$


где $\varepsilon_{i j k}$ - полностью антисимметричный тензор третьего ранга, $\varepsilon_{123}=1$, а подъем и опускание индексов производится с помощью символов Кронекера. Здесь первый индекс $k$ нумерует базис алгебры, а индексы $i, j$ рассматриваются как матричные. Элемент алгебры параметризуется трехмерным вектором $\omega^{k} \in \mathbb{R}^{3}$, и, значит, группа $\mathbb{O}(3)$ является трехмерной. Каждому элементу алгебры соответствует элемент групш $\mathbb{S O}(3)$ (связной компоненты единицы):

$$
S_{(+) i}{ }^{j}=\left(e^{(\omega \varepsilon)}\right)_{i}^{j}=\delta_{i}^{j} \cos \omega+\frac{(\omega \varepsilon)_{i}^{j}}{\omega} \sin \omega+\frac{\omega_{i} \omega^{j}}{\omega^{2}}(1-\cos \omega) \in \mathbb{S O}(3),
$$

где $\omega=\sqrt{\omega^{i} \omega_{i}}-$ модуль вектора $\omega^{i}$. С помощью прямых вычислений нетрудно убедиться, что $S_{(+) i}{ }^{j}$ является действительно ортогональной матрищей. Обратно, любую ортогональную матрицу с положительным определителем можно представить в виде (2) для некоторого вектора $\omega^{i}$. Отметим, что в отличие от элемента алгебры элемент группы имеет как симметричную, так и антисимметричную части. Элемент группы $\mathbb{S} \mathbb{O}(3)$ инвариантен относительно сдвига вектора $\omega^{i} \rightarrow \omega^{i}+2 \pi \omega^{i} / \omega$ :

$$
S_{(+) i}^{j}\left(\omega^{k}+2 \pi \frac{\omega^{k}}{\omega}\right)=S_{(+) i}{ }^{j}\left(\omega^{k}\right),
$$

и это единственная инвариантность. При сдвиге вектора $\omega^{i}$ меняется только его длина $\omega \rightarrow \omega+2 \pi$, а направление остается неизменным. В инвариантности матрищы вращений нетрудно убедиться, если заметить, что отношение $\omega^{i} / \omega$, определяющее направление вектора $\omega^{i}$, остается неизменным при произвольном сдвиге:

$$
\frac{\omega^{i}}{\omega} \rightarrow \frac{\omega^{i}+c \omega^{i} / \omega}{\omega+c}=\frac{\omega^{i}}{\omega}, \quad c \in \mathbb{R} .
$$

Тем самым элемент группы врашений параметризуется точками евклидова пространства $\omega^{i} \in \mathbb{R}^{3}$ с единственным отношением эквивалентности

$$
\omega^{i} \approx \omega^{i}+2 \pi \frac{\omega^{i}}{\omega}
$$

При этом неопределенность в нуле раскрывается по радиальным направлениям $\omega^{i}=$ $\epsilon k^{i}, \epsilon \rightarrow 0$, т.е. начало координат отождествляется со всеми сферами радиуса $2 \pi m$, $m=1,2, \ldots$.

Вектор $\omega^{i}$ параметризует групп $\mathbb{S O}(3)$ следуюшим образом. Направление вектора $\omega^{i}$ совпадает с осью вращения, а модуль вектора $\omega$ равен углу поворота. Таким образом, каждый элемент групшы $\mathbb{S O}(3)$ отождествляется с точкой трехмерного шара $\mathbb{B}_{\pi}^{3}$ радиуса $\pi$ с центром в начале координат. При этом различным внутренним точкам шара соответствуют различные врашения, а диаметрально противоположные точки граничной сферы необходимо отождествить, так как повороты вокруг фиксированной оси на углы $\pi$ и $-\pi$ приводят к одному и тому же результату.

Полная группа трехмерных вращений $\mathbb{O}(3)$ состоит из двух несвязных компонент ортогональных матриц с положительным $S_{(+)}$и отрицательным $S_{(-)}$определителем. 
Элементы полной группы $\mathbb{O}(3)$ параметризуются элементом алгебры (1), например, следующим образом:

$$
S_{( \pm) i}{ }^{j}=\delta_{i}^{j} \cos \omega+\frac{(\omega \varepsilon)_{i}{ }^{j}}{\omega} \sin \omega+\frac{\omega_{i} \omega^{j}}{\omega^{2}}( \pm 1-\cos \omega) \in \mathbb{O}(3) .
$$

Отметим, что каждому элементу алгебры $(\omega \varepsilon)_{i}{ }^{j} \in \mathfrak{s o}(3)$ соответствуют два элемента группы $S_{( \pm)} \in \mathbb{O}(3)$ - по одному из каждой компоненты.

Обратные матрицы имеют вид

$$
S_{( \pm) i}^{-1}{ }^{j}\left(\omega^{k}\right)=S_{( \pm) i}{ }^{j}\left(-\omega^{k}\right)=\delta_{i}^{j} \cos \omega-\frac{(\omega \varepsilon)_{i}{ }^{j}}{\omega} \sin \omega+\frac{\omega_{i} \omega^{j}}{\omega^{2}}( \pm 1-\cos \omega) \in \mathbb{O}(3),
$$

т.е. соответствуют противоположному вектору $-\omega^{i} \in \mathbb{R}^{3}$. Другими словами, обратный элемент группы представляет собой поворот евклидова пространства вокруг той же оси, но на противоположньй угол.

Свернем матрицу врашений $S_{( \pm) i}{ }^{j}$ с вектором $\omega_{j}: S_{( \pm) i}{ }^{j} \omega_{j}= \pm \omega_{i}$. Это значит, что вектор $\omega^{i}$ является собственным вектором матриц врашений с собственными значениями \pm 1 . Другими словами, врашения $S_{(+)}$и $S_{(-)}$соответственно оставляют ось врашения без изменения или меняют ее направление на противоположное.

Допустим, что элементы группы $\mathbb{O}(3)$ гладко зависят от точки произвольного многообразия $\mathbb{M}$, т.е. $\omega^{i}(x): \mathbb{M} \rightarrow \mathbb{O}(3)$. Введем обозначение

$$
l_{( \pm) \alpha i}{ }^{j}=\left(\partial_{\alpha} S_{( \pm)}^{-1} S_{( \pm)}\right)_{i}{ }^{j}
$$

С помошью прямых вычислений можно убедиться в справедливости следуюшей формулы:

$$
\begin{aligned}
l_{( \pm) \alpha i}{ }^{j}= & \mp \frac{\left(\partial_{\alpha} \omega \varepsilon\right)_{i}{ }^{j}}{\omega} \sin \omega-\frac{\partial_{\alpha} \omega(\omega \varepsilon)_{i}{ }^{j}}{\omega}\left(1 \mp \frac{\sin \omega}{\omega}\right) \pm \\
& \pm \frac{\partial_{\alpha} \omega_{i} \omega^{j}-\omega_{i} \partial_{\alpha} \omega^{j}}{\omega^{2}}( \pm 1-\cos \omega) \in \mathfrak{s o}(3) .
\end{aligned}
$$

Эта матрица антисимметрична по своим индексам и, значит, является элементом алгебры $\mathfrak{s o}(3)$. Она представляет собой тривиальную $\mathbb{O}(3)$-связность, для которой тензор кривизны тождественно равен нулю (чистая калибровка). В дальнейшем тривиальную $\mathbb{S O}(3)$-связность будем обозначать $l_{\alpha i}{ }^{j}=l_{(+) \alpha i}{ }^{j}$.

Из приведенной параметризации группы врашений следует, что групповое многообразие компактно и ориентируемо. Как многообразие связная компонента единицы $\mathbb{S} \mathfrak{O}(3)$ диффеоморфна трехмерному проективному пространству $\mathbb{R P}^{3}$. Она не является односвязной: ее фундаментальной группой является $\mathbb{Z}_{2}$. 


\section{3. ЛАГРАНЖИАН МОДЕЛИ СКИРМА}

Рассмотрим $n$-мерное пространство Минковского $\mathbb{R}^{1, n-1}$ с декартовыми координатами $x^{\alpha}, \alpha=0,1, \ldots, n-1$. Пусть в каждой точке пространства Минковского задан элемент группы врашений $\omega^{i}(x)$. Для описания $\pi$-мезонов Скирм предложил следующее действие [1]:

$$
S=\int d x L=\int d x\left(-\frac{\varrho}{4} \operatorname{tr}\left(l_{\alpha} l^{\alpha}\right)-\frac{\kappa}{8} \operatorname{tr}\left(\left[l_{\alpha}, l_{\beta}\right]\left[l^{\alpha}, l^{\beta}\right]\right)\right),
$$

где $\varrho$ и $\kappa$ - константы связи, а квадратные скобки обозначают коммутатор матриц. Скирм рассматривал $l_{\alpha}$ как тривиальную $\mathbb{S U}(2)$-связность и параметризовал ее четырехмерным вектором в изотопическом пространстве, принимающим значения на трехмерной сфере $\mathbb{S}^{3}$, т.е. рассматривал действие $(8)$ как $\mathbb{S} U(2)$-модель или, что эквивалентно, как $\mathbb{S}^{3}$-модель. Мы будем рассматривать действие (8) как действие для $\mathbb{S} \mathfrak{O}(3)$ модели, используя параметризацию группы вращений из предыдущего раздела. Сразу отметим, что эти модели неэквивалентны. Подстановка явного выражения для $l_{\alpha}$ через групповые параметры (7) приводит к следующему лагранжиану для трех независимых скалярных полей $\omega^{i}$ :

$$
\begin{aligned}
L= & \varrho\left(\frac{1}{2} \partial_{\alpha} \omega^{2}+(1-\cos \omega) \frac{\left(\partial_{\alpha} \omega \partial_{\alpha} \omega\right)-\partial_{\alpha} \omega^{2}}{\omega^{2}}\right)+ \\
& +\kappa\left((1-\cos \omega) \frac{\left(\partial_{\alpha} \omega \partial_{\alpha} \omega\right) \partial_{\beta} \omega^{2}-\left(\partial_{\alpha} \omega \partial_{\beta} \omega\right) \partial_{\alpha} \omega \partial_{\beta} \omega}{\omega^{2}}+(1-\cos \omega)^{2} \times\right. \\
& \left.\times \frac{\left(\partial_{\alpha} \omega \partial_{\alpha} \omega\right)^{2}-\left(\partial_{\alpha} \omega \partial_{\beta} \omega\right)^{2}-2\left(\partial_{\alpha} \omega \partial_{\alpha} \omega\right) \partial_{\beta} \omega^{2}+2\left(\partial_{\alpha} \omega \partial_{\beta} \omega\right) \partial_{\alpha} \omega \partial_{\beta} \omega}{\omega^{4}}\right),
\end{aligned}
$$

где введено обозначение для скалярного произведения

$$
\left(\partial_{\alpha} \omega \partial_{\alpha} \omega\right)=\partial_{\alpha} \omega^{i} \partial_{\alpha} \omega_{i} \neq \partial_{\alpha} \omega^{2}
$$

В этом выражении по повторяюшимся греческим индексам подразумевается суммирование с метрикой Минковского $\eta_{\alpha \beta}$, и мы для простоты не делаем различия между нижними и верхними индексами. Три поля $\omega^{i}(x)$, от которых зависит лагранжиан, являются независимыми динамическими переменными, на которые не наложены никакие дополнительные условия, кроме отношения эквивалентности (3). Эти поля являются скалярами относительно преобразования координат в пространстве Минковского $\mathbb{R}^{1, n-1}$ и компонентами вектора относительно глобальных вращений евклидова пространства-мишени $\mathbb{R}^{3}$. Можно проверить, что лагранжиан (9) инвариантен относительно группы Пуанкаре, действующей в пространстве Минковского, глобальных $\mathbb{O}(3)$ вращений, действующих в пространстве-мишени $\omega^{i} \in \mathbb{R}^{3}$, и локальных сдвигов (3). Последнее преобразование означает следующее. Длину вектора $\omega^{i} \in \mathbb{R}^{3}$ в каждой точке $x \in \mathbb{R}^{1, n-1}$ можно менять на постоянную величину, кратную $2 \pi$. В то же время это дискретное преобразование является локальным, поскольку направление вектора $\omega^{i}$ меняется от точки к 
точке. В силу этой инвариантности мы будем считать, что вектор $\omega^{i}$ лежит внутри шара радиуса $\pi$ с центром в начале координат пространства-мишени $\omega^{i} \in \mathbb{B}_{\pi}^{3} \subset \mathbb{R}^{3}$.

Для исследования модели Скирма в виде (9) вместо компонент $\omega^{i}$ удобно рассматривать четыре новые переменные: длину вектора $\omega^{i}$ (угол поворота $\omega$ ) и его направление (ось врашения $k^{i}$ )

$$
\omega=\sqrt{\left(\omega^{1}\right)^{2}+\left(\omega^{2}\right)^{2}+\left(\omega^{3}\right)^{2}}, \quad k^{i}=\frac{\omega^{i}}{\omega} .
$$

По определению вектор $k^{i}$ имеет единичную длину: $(k, k)=k^{i} k_{i}=1$. В терминах новых переменных лагранжиан (9) принимает вид

$$
\begin{aligned}
L= & \varrho\left(\frac{1}{2} \partial_{\alpha} \omega^{2}+(1-\cos \omega)\left(\partial_{\alpha} k \partial_{\alpha} k\right)\right)+ \\
& +\kappa\left((1-\cos \omega)\left[\left(\partial_{\alpha} k \partial_{\alpha} k\right) \partial_{\beta} \omega^{2}-\left(\partial_{\alpha} k \partial_{\beta} k\right) \partial_{\alpha} \omega \partial_{\beta} \omega\right]+\right. \\
& \left.+(1-\cos \omega)^{2}\left[\left(\partial_{\alpha} k \partial_{\alpha} k\right)^{2}-\left(\partial_{\alpha} k \partial_{\beta} k\right)^{2}\right]\right) .
\end{aligned}
$$

Напомним, что $\left(\partial_{\alpha} k \partial_{\alpha} k\right)=\partial_{\alpha} k^{i} \partial_{\alpha} k_{i}$. Преобразование (3) меняет только длину вектоpa:

$$
\omega \rightarrow \omega+2 \pi, \quad k^{i} \rightarrow k^{i} .
$$

Отсюда сразу следует инвариантность лагранжиана (11) относительно преобразований (12). Более того, инвариантным является каждое слагаемое само по себе.

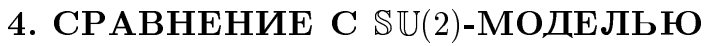

Вид действия модели Скирма (8) для групповых параметров зависит только от алгебры, но не от группы, которая является пространством-мишенью. Чтобы продемонстрировать зависимость модели от группы, рассмотрим $\mathbb{S} U(2)$-модель. Элемент алгебры $\mathfrak{s u}(2)$ имеет вид

$$
\omega^{k} \frac{i}{2} \sigma_{k \mathrm{~A}}{ }^{\mathrm{B}} \in \mathfrak{s u}(2), \quad \mathrm{A}, \mathrm{B}=1,2,
$$

где $\sigma_{k}$ - матрицы Паули. Множитель $i / 2$ выбран для того, чтобы коммутаторы векторов базиса имели одинаковый вид для алгебр $\mathfrak{s u}(2)$ и $\mathfrak{s o}(3)$. Соответствуюший элемент группы $\mathbb{S U}(2)$ имеет вид

$$
U_{\mathrm{A}}^{\mathrm{B}}=\left(e^{i \omega \sigma / 2}\right)_{\mathrm{A}}{ }^{\mathrm{B}}=\delta_{\mathrm{A}}^{\mathrm{B}} \cos \frac{\omega}{2}+i \frac{\omega^{k} \sigma_{k \mathrm{~A}}{ }^{\mathrm{B}}}{\omega} \sin \frac{\omega}{2} \in \mathbb{S U}(2) .
$$

Из вида элемента группы следует равенство

$$
U_{\mathrm{A}}^{\mathrm{B}}\left(\omega^{i}+4 \pi \frac{\omega^{i}}{\omega}\right)=U_{\mathrm{A}}^{\mathrm{B}}\left(\omega^{i}\right),
$$

т.е. для группы $\mathbb{S} \mathbb{U}(2)$ в пространстве параметров имеется отношение эквивалентности

$$
\omega^{i} \approx \omega^{i}+4 \pi \frac{\omega^{i}}{\omega}
$$


По сравнению с отношением эквивалентности (3) для группы $\mathbb{S} \mathfrak{O}(3)$ сдвиг происходит на вектор вдвое большей длины. Тем самым групповое многообразие параметризуется внутренними точками шара $\mathbb{B}_{2 \pi}^{3}$ радиуса $2 \pi$. При этом все точки граничной сферы необходимо отождествить. Это является вторым отношением эквивалентности в пространстве параметров:

$$
\left.\left.\omega_{1}^{i}\right|_{\omega_{1}=2 \pi} \approx \omega_{2}^{i}\right|_{\omega_{2}=2 \pi}
$$

Дополнительное соотношение эквивалентности связано с отсутствием третьего слагаемого в (13) по сравнению с (2). В этом также есть отличие от группы врашений, для которой отождествляются только диаметрально противоположные точки граничной сферы, что входит в отношение эквивалентности (3). Можно показать, что в пространстве параметров для группы $\mathbb{S} \mathbb{U}(2)$ других отношений эквивалентности, кроме (14) и (15), не существует.

Нетрудно проверить, что лагранжиан модели Скирма имеет один и тот же вид (11) для обеих групп $\mathbb{S} \mathbb{U}(2)$ и $\mathbb{S O}(3)$. Разница сводится к отношениям эквивалентности (3) и (14) и отождествлению точек граничной сферы (15). В силу последнего обстоятельства возникает отличие в фундаментальных группах, что приводит к существованию топологически-нетривиальных одномерных решений в $\mathbb{S}(3)$-модели Скирма.

\section{5. $\mathbb{S}^{2}$-МОДЕЛЬ}

В течение многих лет большое внимание привлекает $\mathbb{S}^{2}$-модель. Поскольку $\mathbb{S O}(3)$-модель Скирма и $\mathbb{S}^{2}$-модель описываются трехмерными векторами в пространстве-мишени, и любой вектор заданной длины можно представить как результат действия матрицы вращений на фиксированный вектор той же длины, то возникает вопрос о связи этих моделей. Несмотря на то что модели неэквивалентны, между ними существует связь, которую мы рассмотрим в настоящем разделе.

Свяжем $\mathbb{S O}(3)$-модель из раздела $3 \mathrm{c} \mathbb{S}^{2}$-моделью. Пусть в каждой точке пространства Минковского $\mathbb{R}^{1, n-1}$ задан трехмерный единичный вектор $n^{i}(x),(n, n)=1$. Это значит, что пространством-мишенью является двумерная сфера $\mathbb{S}^{2}$. Декартовы координаты в пространстве-мишени будем обозначать заглавными буквами $X, Y, Z$. Рассмотрим лагранжиан, предложенный Фаддеевым для $\mathbb{S}^{2}$-модели [6]:

$$
\begin{aligned}
L_{\mathrm{F}} & =m^{2}\left(\partial_{\alpha} n \partial_{\alpha} n\right)+\frac{1}{e^{2}}\left(n^{i} \varepsilon_{i j k} \partial_{\alpha} n^{j} \partial_{\beta} n^{k}\right)^{2}= \\
& =m^{2}\left(\partial_{\alpha} n \partial_{\alpha} n\right)+\frac{1}{e^{2}}\left[\left(\partial_{\alpha} n \partial_{\alpha} n\right)^{2}-\left(\partial_{\alpha} n \partial_{\beta} n\right)^{2}\right] .
\end{aligned}
$$

Эта модель имеет нетривиальные топологические решения, связанные с третьей гомотопической группой $\pi_{3}\left(\mathbb{S}^{2}\right)=\mathbb{Z}[7]$.

Произвольный единичный вектор $n \in \mathbb{S}^{2}$ можно параметризовать элементом группы трехмерных вращений

$$
n^{i}=n_{0}^{j} S_{j}^{i}(\omega)
$$


где $n_{0}^{i}$ - произвольный фиксированный вектор единичной длины. Это соответствие не является взаимно однозначным, так как два заданных вектора $n$ и $n_{0}$ определяют элемент группы врашений неоднозначно. Забегая вперед, скажем, что $\mathbb{S} O(3)-$ и $\mathbb{S}^{2}$-модели неэквивалентны. При параметризации (17) лагранжиан (16) примет вид

$$
\begin{aligned}
L_{\mathrm{F}}= & m^{2} n_{0}^{i} n_{0}^{j} \partial_{\alpha} S_{i}{ }^{k} \partial_{\alpha} S_{j k}+ \\
& +\frac{1}{e^{2}} n_{0}^{i} n_{0}^{j} n_{0}^{k} n_{0}^{l}\left(\partial_{\alpha} S_{i}{ }^{m} \partial_{\alpha} S_{j m} \partial_{\beta} S_{k}{ }^{n} \partial_{\beta} S_{l n}-\partial_{\alpha} S_{i}{ }^{m} \partial_{\beta} S_{j m} \partial_{\beta} S_{k}{ }^{n} \partial_{\alpha} S_{l n}\right) .
\end{aligned}
$$

Он зависит от трех скалярных полей $\omega^{i}(x)$, параметризующих группу вращений, и фиксированного вектора $n_{0}$. Усредним действие по всем углам, определяющим вектор $n_{0}$. С этой целью воспользуемся формулами усреднения, которые проверяются простыми вычислениями:

$$
\begin{aligned}
\left\langle n_{0}^{i} n_{0}^{j}\right\rangle & =\frac{1}{4 \pi} \int d \Theta d \Phi \sin \Theta n_{0}^{i} n_{0}^{j}=\frac{1}{3} \delta^{i j} \\
\left\langle n_{0}^{i} n_{0}^{j} n_{0}^{k} n_{0}^{l}\right\rangle & =\frac{1}{4 \pi} \int d \Theta d \Phi \sin \Theta n_{0}^{i} n_{0}^{j} n_{0}^{k} n_{0}^{l}=\frac{1}{15}\left(\delta^{i j} \delta^{k l}+\delta^{i k} \delta^{j l}+\delta^{i l} \delta^{j k}\right),
\end{aligned}
$$

где $\Theta, \Phi$ - полярные углы в пространстве-мишени. После усреднения получим новый лагранжиан

$$
\left\langle L_{\mathrm{F}}\right\rangle=-\frac{m^{2}}{3} \operatorname{tr}\left(l_{\alpha} l_{\alpha}\right)-\frac{1}{15 e^{2}}\left[\frac{1}{2} \operatorname{tr}\left(\left[l_{\alpha}, l_{\beta}\right]\left[l_{\alpha}, l_{\beta}\right]\right)-\operatorname{tr}^{2}\left(l_{\alpha} l_{\alpha}\right)+\operatorname{tr}^{2}\left(l_{\alpha} l_{\beta}\right)\right]
$$

Он содержит два дополнительных члена помимо слагаемых модели Скирма (8).

Таким образом, усреднение $\mathbb{S}^{2}$-модели по направлениям вектора $n_{0}$ приводит к $\mathbb{S} \mathbb{O}(3)$ модели. Неэквивалентность моделей будет видна на примере статических решений, рассмотренных в следующих разделах.

\section{6. СТАТИЧЕСКИЕ РЕШЕНИЯ В ДВУМЕРНОЙ $\mathbb{S}^{2}$-МОДЕЛИ}

Единичный вектор $n$ в сферической системе координат параметризуется двумя углами $\Theta$ и $\Phi:$

$$
n^{1}=\sin \Theta \cos \Phi, \quad n^{2}=\sin \Theta \sin \Phi, \quad n^{3}=\cos \Theta,
$$

которые описывают две независимые степени свободы. Для простоты мы будем исследовать статические решения в двумерной $\mathbb{S}^{2}$-модели. В этом случае второе слагаемое в лагранжиане (16) не дает вклада в уравнения для статических решений. Поэтому мы ограничимся рассмотрением только первого слагаемого.

В угловых координатах действие, соответствуюшее первому слагаемому в (16), принимает вид

$$
S=m^{2} \int d x\left(\partial_{\alpha} \Theta^{2}+\sin ^{2} \Theta \partial_{\alpha} \Phi^{2}\right)
$$


и приводит к уравнениям движения

$$
\begin{aligned}
& \frac{1}{m^{2}} \frac{\delta S}{\delta \Theta}=-2 \square \Theta+2 \sin \Theta \cos \Theta \partial_{\alpha} \Phi^{2}=0, \\
& \frac{1}{m^{2}} \frac{\delta S}{\delta \Phi}=-2 \partial_{\alpha}\left(\partial_{\alpha} \Phi \sin ^{2} \Theta\right)=0
\end{aligned}
$$

где $\square=\partial_{\alpha} \partial_{\alpha}-$ оператор Д'Аламбера.

Рассмотрим двумерное пространство Минковского $\mathbb{R}^{1,1}$ с декартовыми координатами $t, x$ и проанализируем статические решения. Для $\Theta=\Theta(x)$ и $\Phi=\Phi(x)$ уравнения движения (20), (21) принимают вид

$$
\begin{aligned}
\Theta^{\prime \prime}-\sin \Theta \cos \Theta \Phi^{\prime 2} & =0 \\
\left(\Phi^{\prime} \sin ^{2} \Theta\right)^{\prime} & =0
\end{aligned}
$$

где штрих означает дифференцирование по $x$. Будем искать решение системы обыкновенных дифференциальных уравнений в классе дважды непрерывно дифференцируемых функций $\mathcal{C}^{2}(L)$ на конечном отрезке $x \in[0, L]$. Любое решение этой системы уравнений принадлежит одному из следующих четырех классов (постоянные интегрирования ниже обозначены через $b, c, x_{0}$ и $\left.\Phi_{0}\right)$.

\section{Вырожденные решения I.}

$$
\Theta=0, \quad \Phi(x) \in C^{2}(L) .
$$

В этом случае вектор $n$ направлен вдоль оси $Z$, и никакого врашения не происходит.

\section{Вырожденные решения II.}

$$
\Theta=\frac{\pi}{2}, \quad \Phi=b x+x_{0}
$$

При этом вектор $n$ по мере движения вдоль $x$ равномерно врашается в плоскости $X, Y$.

\section{Вырожденные решения III.}

$$
\Theta=b x+x_{0}, \quad \Phi=\Phi_{0} .
$$

При этом вектор $n$ по мере движения вдоль $x$ равномерно врашается в плоскости $\Phi=\Phi_{0}$. Эти решения по сути дела совпадают с вырожденными решениями II. Чтобы показать это, воспользуемся свободой в выборе координат в пространстве-мишени и выберем новую ось $Z^{\prime}$ перпендикулярно плоскости $\Phi=\Phi_{0}$. Тогда в новой системе координат вырожденные решения III перейдут в вырожденные решения II. 
Общее положение. В этом случае уравнение (23) имеет решение

$$
\Phi^{\prime} \sin ^{2} \Theta=c, \quad c \neq 0
$$

Тогда уравнение (22) принимает вид

$$
\Theta^{\prime \prime}=c^{2} \frac{\cos \Theta}{\sin ^{3} \Theta} .
$$

Оно легко интегрируется:

$$
\cos \Theta=\sqrt{1-\frac{c^{2}}{b^{2}}} \sin \left[b\left(x+x_{0}\right)\right], \quad|b|>|c| .
$$

После этого интегрируем уравнение (27):

$$
\operatorname{tg}\left(\Phi+\Phi_{0}\right)=\frac{c}{b} \operatorname{tg}\left[b\left(x+x_{0}\right)\right] .
$$

Таким образом, формулы (28), (29) дают статические решения обшего положения. Постоянные интегрирования $x_{0}$ и $\Phi_{0}$ соответствуют сдвигам координат, и для удобства их можно положить равными нулю. Постоянная $b$ соответствует выбору масштаба координаты $x$. При фиксированной длине отрезка $L$ его менять нельзя. Отметим, что вырожденные решения II получаются из решений обшего положения в пределе $|b|=|c|$.

Можно проверить, что в случае общего положения вектор $n$ по мере движения вдоль $x$ равномерно врашается в плоскости, наклоненной к плоскости $X, Y$ под углом $\arccos (c / b)$. Это значит, что по сути дела имеется только два сушественно различных решения уравнений Эйлера-Лагранжа. Это, во-первых, вырожденные решения I, когда вектор $n$ не меняется по мере движения вдоль $x$. Во-вторых, остальные решения описывают равномерное вращение вектора $n$ в произвольной, но фиксированной плоскости. В этом случае ось врашения всегда перпендикулярна вектору $n$. Деление этих решений на вырожденные и решения обшего положения связано с ориентацией системы координат относительно плоскости врашения. Отметим, что во всех случаях ориентация декартовых осей $X, Y, Z$ в пространстве-мишени может быть произвольной по отношению к пространственной координате $x$.

Все найденные решения $\mathbb{S}^{2}$-модели гомотопны нулю, так как фундаментальная группа тривиальна: $\pi\left(\mathbb{S}^{2}\right)=0$. Это значит, что в $\mathbb{S}^{2}$-моделях одномерные топологическинетривиальные решения отсутствуют.

Энергия двумерной $\mathbb{S}^{2}$-модели для статических решений равна

$$
E=\int_{0}^{L} d x\left(\Theta^{\prime 2}+\sin ^{2} \Theta \Phi^{\prime 2}\right) .
$$

Прямая подстановка решений в этот интеграл приводит к следуюшему значению:

$$
E= \begin{cases}0, & \text { вырожденные решения I, } \\ b^{2} L, & \text { остальные решения. }\end{cases}
$$


Тем самым энергия статических решений конечна при конечных $L$. При $b L=2 \pi m, m=$ $1,2, \ldots$, решения периодичны. В этом случае их можно рассматривать как заданные на окружности.

Проанализируем устойчивость полученных решений. Вторая вариация энергии имеет вид

$$
\delta^{2} E=\int_{0}^{L} d x\left(\delta \Theta^{\prime 2}+\cos (2 \Theta) \Phi^{\prime 2} \delta \Theta^{2}+2 \sin (2 \Theta) \Phi^{\prime} \delta \Theta \delta \Phi^{\prime}+\sin ^{2} \Theta \delta \Phi^{\prime 2}\right) .
$$

В этом выражении вариации $\delta \Theta, \delta \Theta^{\prime}, \delta \Phi$ и $\delta \Phi^{\prime}$ следует считать независимыми функциями. Простой анализ показывает, что вырожденные решения I дают абсолютный минимум энергии, а остальные решения соответствуют седловым точкам.

\section{7. СТАТИЧЕСКИЕ РЕШЕНИЯ

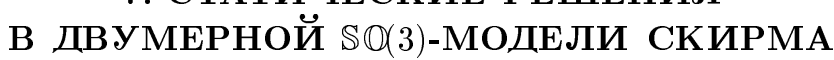

Уравнения Эйлера-Лагранжа для $\mathbb{S} \mathfrak{O}(3)$-модели Скирма (11) имеют вид

$$
\begin{aligned}
\frac{\delta S}{\delta \omega}= & -\varrho \square \omega+\varrho \sin \omega\left(\partial_{\alpha} k \partial_{\alpha} k\right)-2 \kappa(1-\cos \omega) \times \\
& \times\left[\left(\partial_{\alpha \beta}^{2} k \partial_{\alpha} k\right) \partial_{\beta} \omega+\left(\partial_{\alpha} k \partial_{\alpha} k\right) \square \omega-\left(\partial_{\alpha} k \square k\right) \partial_{\alpha} \omega-\left(\partial_{\alpha} k \partial_{\beta} k\right) \partial_{\alpha} \omega \partial_{\beta} \omega\right]- \\
& -\kappa \sin \omega\left[\left(\partial_{\alpha} k \partial_{\alpha} k\right) \partial_{\beta} \omega^{2}-\left(\partial_{\alpha} k \partial_{\beta} k\right) \partial_{\alpha} \omega \partial_{\beta} \omega\right]+ \\
& +2 \kappa(1-\cos \omega) \sin \omega\left[\left(\partial_{\alpha} k \partial_{\alpha} k\right)^{2}-\left(\partial_{\alpha} k \partial_{\beta} k\right)^{2}\right]=0, \\
\frac{\delta S}{\delta k_{i}}= & -2 \varrho(1-\cos \omega)\left[\square k^{i}+\left(\partial_{\alpha} k \partial_{\alpha} k\right) k^{i}\right]-2 \varrho \sin \omega \partial_{\alpha} k^{i} \partial_{\alpha} \omega- \\
& -2 \kappa(1-\cos \omega)\left[\square k^{i} \partial_{\alpha} \omega^{2}+\partial_{\alpha} k^{i} \partial_{\alpha \beta}^{2} \omega \partial_{\beta} \omega-\partial_{\alpha \beta}^{2} k^{i} \partial_{\alpha} \omega \partial_{\beta} \omega-\partial_{\alpha} k^{i} \square \omega \partial_{\alpha} \omega\right]- \\
& -4 \kappa(1-\cos \omega)^{2} \times \\
& \times\left[\left(\partial_{\alpha} k \partial_{\alpha} k\right) \square k^{i}+\left(\partial_{\alpha \beta}^{2} k \partial_{\beta} k\right) \partial_{\alpha} k^{i}-\left(\square k \partial_{\alpha} k\right) \partial_{\alpha} k^{i}-\left(\partial_{\alpha} k \partial_{\beta} k\right) \partial_{\alpha \beta}^{2} k^{i}\right]- \\
& -8 \kappa(1-\cos \omega) \sin \omega\left[\left(\partial_{\beta} k \partial_{\beta} k\right) \partial_{\alpha} k^{i}-\left(\partial_{\alpha} k \partial_{\beta} k\right) \partial_{\beta} k^{i}\right] \partial_{\alpha} \omega- \\
& -2 \kappa(1-\cos \omega)\left[\left(\partial_{\alpha} k \partial_{\alpha} k\right) \partial_{\beta} \omega^{2}-\left(\partial_{\alpha} k \partial_{\beta} k\right) \partial_{\alpha} \omega \partial_{\beta} \omega\right] k^{i}- \\
& -4 \kappa(1-\cos \omega)^{2}\left[\left(\partial_{\alpha} k \partial_{\alpha} k\right)^{2}-\left(\partial_{\alpha} k \partial_{\beta} k\right)^{2}\right] k^{i}=0 .
\end{aligned}
$$

При варьировании действия по единичному вектору $k_{i}$ мы воспользовались формулой, учитываюшей связь $(k, k)=1$ :

$$
\frac{\delta S}{\delta k_{i}}=\frac{\bar{\delta} S}{\bar{\delta} k_{j}}\left(\delta_{j}^{i}-k^{i} k_{j}\right)
$$

где вариационная производная в правой части берется без учета связи.

Нетрудно убедиться, что для статических решений $\omega(x), k^{i}(x)$ в двумерном пространстве Минковского $\mathbb{R}^{1,1}$ все слагаемые, зависящие от константы связи $\kappa$, сокрашаются. Это значит, что для исследования одномерных статических решений в $\mathbb{S} \mathfrak{O}(3)$-модели достаточно рассмотреть лагранжиан

$$
L=\varrho\left[\frac{1}{2} \partial_{\alpha} \omega^{2}+(1-\cos \omega)\left(\partial_{\alpha} \theta^{2}+\sin ^{2} \theta \partial_{\alpha} \varphi^{2}\right)\right],
$$


где использована сферическая система координат в пространстве-мишени:

$$
k^{1}=\sin \theta \cos \varphi, \quad k^{2}=\sin \theta \sin \varphi, \quad k^{3}=\cos \theta .
$$

Чтобы отличить единичный вектор $k$ от вектора $n$, рассмотренного в предыдушем разделе, мы используем строчные греческие буквы для обозначения угловых координат вектора $k$.

Уравнения движения в статическом случае принимают вид

$$
\begin{aligned}
& \frac{1}{\varrho} \frac{\delta S}{\delta \omega}=-\omega^{\prime \prime}+\sin \omega\left(\theta^{2}+\sin ^{2} \theta \varphi^{\prime 2}\right)=0, \\
& \frac{1}{\varrho} \frac{\delta S}{\delta \theta}=-2\left((1-\cos \omega) \theta^{\prime}\right)^{\prime}+2(1-\cos \omega) \sin \theta \cos \theta \varphi^{\prime 2}=0, \\
& \frac{1}{\varrho} \frac{\delta S}{\delta \varphi}=-2\left((1-\cos \omega) \sin ^{2} \theta \varphi^{\prime}\right)^{\prime}=0 .
\end{aligned}
$$

Будем искать решение этой системы дифференциальных уравнений в классе функций $\mathcal{C}^{2}(L)$. Любое решение системы уравнений $(34)-(36)$ принадлежит одному из следуюших пяти классов (константы интегрирования обозначены ниже через $a, b, c, x_{0}, \varphi_{0}, \theta_{0}$ и $\left.u_{0}\right)$.

Вырожденные решения I. В этом случае угол врашения является линейной функцией

$$
\omega=b x+x_{0}
$$

а ось вращения остается неподвижной:

$$
\theta=\theta_{0}, \quad \varphi=\varphi_{0}, \quad \text { или } \quad \theta=0, \quad \varphi(x) \in \mathcal{C}^{2}(L) .
$$

Для этого решения вектор $n^{i}=n_{0}^{j} S_{j}{ }^{i}(\omega)$ при движении вдоль $x$ равномерно вращается вокруг вектора $k$, имеющего произвольное направление. В отличие от $\mathbb{S}^{2}$-модели, вектор $n$ не обязательно перпендикулярен оси вращения.

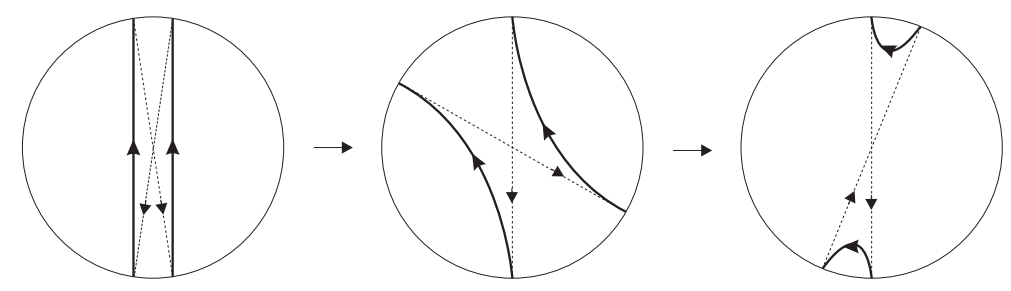

Вырожденные решения I дают пример статических топологически-нетривиальных решений с топологическим зарядом 0 или 1. Если угол $\omega$ меняется в интервале, кратном $4 \pi$, то топологический заряд равен 0 , так как соответствуюший контур всегда может быть непрерывно деформирован в точку. На рисунке показана соответствующая непрерывная деформация замкнутого контура при $0<\omega<4 \pi$ в точку. Деформация показана в плоскости, проходящей через вектор $k$, определяющий направление врашения. Пунктиром показано отождествление противоположных точек граничной окружности. Если же угол $\omega$ принимает значения в интервале, содержашем $2 \pi$ нечетное число раз, то топологический заряд такого решения равен 1. 
Вырожденные решения II.

$$
\omega=0, \quad \theta(x), \varphi(x) \in \mathcal{C}^{2}(L)
$$

Для этой серии решений вращения не происходит, так как угол врашения равен нулю.

\section{Вырожденные решения III.}

$$
\begin{gathered}
\cos \frac{\omega}{2}=\sqrt{1-\frac{c^{2}}{4 b^{2}}} \sin \left[b\left(x+x_{0}\right)\right], \quad 2|b| \geqslant|c| \neq 0, \\
\theta=\frac{\pi}{2}, \\
\operatorname{tg}\left(\varphi+\varphi_{0}\right)=\frac{c}{2 b} \operatorname{tg}\left[b\left(x+x_{0}\right)\right] .
\end{gathered}
$$

Для этих решений полярный угол $\varphi$ является монотонной функцией $x$, т.е. ось врашения $k^{i}$ врашается в плоскости $X, Y$. При этом угол врашения меняется в конечном интервале

$$
2 \arccos \sqrt{1-\frac{c^{2}}{4 b^{2}}}<\omega<2 \pi-2 \arccos \sqrt{1-\frac{c^{2}}{4 b^{2}}} .
$$

Нетрудно проверить, что эти решения гомотопны нулю.

\section{Вырожденные решения IV.}

$$
\begin{gathered}
\cos \frac{\omega}{2}=\sqrt{1-\frac{c^{2}}{4 b^{2}}} \sin \left[b\left(x+x_{0}\right)\right], \quad 2|b| \geqslant|c| \neq 0, \\
\operatorname{tg}\left(\theta+\theta_{0}\right)=\frac{c}{2 b} \operatorname{tg}\left[b\left(x+x_{0}\right)\right], \\
\varphi=\varphi_{0} .
\end{gathered}
$$

Эти решения по сути совпадают с вырожденными решениями III, только ось врашения врашается в плоскости $\varphi=\varphi_{0}$.

Общее положение. Для решения системы уравнений (34)-(36) в случае обшего положения введем новую координату

$$
u=\int^{x} \frac{d y}{1-\cos \omega(y)} .
$$

Поскольку $d x / d u=1-\cos \omega \geqslant 0$, то координата $u$ увеличивается с ростом $x$. Тогда уравнения для $\theta$ и $\varphi(35),(36)$ в точности совпадут с уравнениями для $\Theta$ и $\Phi(22),(23)$, в которых дифференцирование по $x$ нужно заменить дифференцированием по $u$. Поскольку эти уравнения были проанализированы в предыдушем разделе, то нам остается только решить уравнение (34). В случае общего положения оно принимает вид

$$
\omega^{\prime \prime}=c^{2} \frac{\sin \omega}{(1-\cos \omega)^{2}}, \quad c \neq 0 .
$$


Обшее решение этого уравнения имеет вид

$$
\cos \frac{\omega}{2}=\sqrt{1-\frac{c^{2}}{4 b^{2}}} \sin \left[b\left(x+x_{0}\right)\right], \quad 2|b| \geqslant|c| .
$$

После этого интеграл (38) легко берется:

$$
\operatorname{tg}(c u)=\frac{c}{2 b} \operatorname{tg}\left[b\left(x+x_{0}\right)\right]
$$

где постоянная интегрирования несушественна. Решение уравнений $(35),(36)$ имеет вид

$$
\begin{gathered}
\cos \theta=\sqrt{1-\frac{a^{2}}{c^{2}}} \sin \left[c\left(u+u_{0}\right)\right], \quad|c|>|a| \neq 0, \\
\operatorname{tg}\left(\varphi+\varphi_{0}\right)=\frac{a}{c} \operatorname{tg}\left[c\left(u+u_{0}\right)\right] .
\end{gathered}
$$

Простой геометрический анализ показывает, что решения общего положения по существу совпадают с вырожденными решениями III и IV, только ось врашения врашается в плоскости, наклоненной под углом $\arccos (a / c)$ к плоскости $X, Y$.

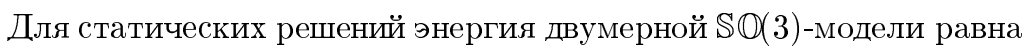

$$
E=\int_{0}^{L} d x\left(\frac{1}{2} \omega^{\prime 2}+(1-\cos \omega)\left(\theta^{\prime 2}+\sin ^{2} \theta \varphi^{\prime 2}\right)\right)
$$

Прямая подстановка решений в этот интеграл приводит к следуюшим значениям:

$$
E= \begin{cases}0, & \text { вырожденные решения II, } \\ \frac{1}{2} b^{2} L, & \text { вырожденные решения I, } \\ 2 b^{2} L, & \text { остальные решения. }\end{cases}
$$

Энергия конечна при конечных $L$. Поскольку энергия положительно определена, то вырожденные решения II соответствуют абсолютному минимуму.

\section{8. ПРОЕКТИВНЫЕ $\mathbb{R P}^{d}-$ МОДЕЛИ}

Лагранжиан модели Скирма, записанный в виде (11), можно естественным образом обобщить на случай, когда пространством-мишенью является вещественное проективное пространство произвольной размерности $\mathbb{R P}^{d}$. Пусть $k^{i}, i=1, \ldots, d,-$ произвольный единичный вектор $\left(k^{2}=1\right)$ евклидова пространства $\mathbb{R}^{d}$. Рассмотрим изотопическое векторное $k^{i}(x)$ и скалярное $\omega(x)$ поля на произвольном пространстве Минковского $x \in \mathbb{R}^{1, n-1}$. Очевидно, что лагранжиан

$$
\begin{aligned}
L= & A \partial_{\alpha} \omega^{2}+B\left(\partial_{\alpha} k \partial_{\alpha} k\right)+C\left(\partial_{\alpha} k \partial_{\alpha} k\right) \partial_{\beta} \omega^{2}+D\left(\partial_{\alpha} k \partial_{\beta} k\right) \partial_{\alpha} \omega \partial_{\beta} \omega+ \\
& +E\left(\partial_{\alpha} k \partial_{\alpha} k\right)^{2}+F\left(\partial_{\alpha} k \partial_{\beta} k\right)^{2}+G\left(\partial_{\alpha} \omega^{2}\right)^{2}+U,
\end{aligned}
$$


где $A(\omega), B(\omega), C(\omega), D(\omega), E(\omega), F(\omega), G(\omega), U(\omega)$ - произвольные периодические с периодом $2 \pi$ функции от $\omega$, инвариантен относительно преобразований (12). В этом лагранжиане так же, как и в модели Скирма, можно перейти к изотопическому векторному полю $\omega^{i}=\omega k^{i}$, все компоненты которого являются независимыми. В силу отношения эквивалентности (3) можно считать, что изотопическое векторное поле $\omega^{i}$ принимает значения внутри шара радиуса $\pi$, у которого отождествлены диаметрально противоположные точки граничной сферы. Это означает, что поля $\omega^{i}$ принимают значения в произвольном проективном пространстве $\mathbb{R P}^{d}$.

Это наиболее общий инвариантный лагранжиан, зависящий только от первых производных полей в степени не выше четвертой. Он инвариантен относительно группы Пуанкаре $\mathbb{I} O(1, n-1)$, действуюшей в пространстве Минковского $\mathbb{R}^{1, n-1}$, группы вращений $\mathbb{O}(d)$, действуюшей в пространстве-мишени $\mathbb{R}^{d}$, и сдвигов (12). Группу врашений $\mathbb{O}(d)$ можно расширить до группы $\mathbb{O}(d+1)$ (максимальной группы симметрии проективного пространства $\left.\mathbb{R P}^{d}\right)$, однако дополнительные преобразования будут действовать на поля $\omega^{i}$ нелинейно.

\section{9. ЗАКЛЮЧЕНИЕ}

Используя явную параметризацию группы $\mathbb{S O}(3)$ элементами ее алгебры, мы рассмотрели модель Скирма как $\mathbb{S} O(3)$-модель, пространством-мишенью которой является само групповое многообразие. Поскольку фундаментальная группа группы вращений нетривиальна, $\pi(\mathbb{S} \mathfrak{O}(3))=\mathbb{Z}_{2}$, то модель допускает сушествование одномерных топологически-нетривиальных решений. Соответствуюшие статические решения в двумерной модели Скирма найдены и проанализированы (вырожденные решения I из раздела 7).

Вид действия для $\mathbb{S} U(2)-$ и $\mathbb{S} \mathbb{O}(3)$-моделей Скирма один и тот же, поскольку совпадают их алгебры Ли. Отличие сводится к отношениям эквивалентности в пространстве параметров. Поэтому при построении моделей математической физики помимо действия необходимо также указывать, какое многообразие является пространством-мишенью. И это важно, так как возникают различные топологически-нетривиальные решения уравнений Эйлера-Лагранжа. Например, при рассмотрении спиноров на трехмерном римановом многообразии в ковариантную производную входит $\mathbb{S O}(3)$-связность, несмотря на то что спиноры преобразуются по представлению унитарной группы $\mathbb{S} U(2)$. Это связано с тем, что в пространстве, касательном к риманову многообразию, на ортогональный репер действует группа $\mathbb{S} \mathbb{O}(3)$, а не $\mathbb{S} \mathbb{U}(2)$.

Поскольку группа $\mathbb{S O}(3)$ как многообразие диффеоморфна трехмерному действительному проективному пространству $\mathbb{R P}^{3}$, то $\mathbb{S} \mathfrak{O}(3)$-модель допускает естественное обобщение на проективные пространства произвольной размерности $\mathbb{R P}^{d}$. Это позволило определить новый класс проективных моделей, зависящий от восьми произвольных периодических функций одного аргумента. Поскольку $\pi\left(\mathbb{R P P}^{d}\right)=\mathbb{Z}_{2}$ при $d \geqslant 2$, то во всех этих моделях возможно сушествование одномерных топологически-нетривиальных решений.

$\mathbb{S O}(3)$-модель может иметь применение в геометрической теории дефектов [8]. Для описания статического распределения дислокаций и дисклинаций в упругой среде ис- 
пользуется геометрия Римана-Картана. Соответствующее действие инвариантно относительно общих преобразований координат и локальных $\mathbb{S O}(3)$-вращений. Недавно уравнения теории упругости было предложено использовать для фиксирования системы координат [9]. Это позволило включить классическую теорию упругости в геометрический подход как предельный случай отсутствия дефектов. Поскольку $\mathbb{S}(3)$-модель, рассмотренная в настоящей статье, неинвариантна относительно локальных $\mathbb{S} \mathbb{O}(3)$-вращений, то ее можно использовать для фиксирования этой инвариантности. В этом случае при отсутствии дисклинаций геометрическая теория дефектов будет приводить к $\mathbb{S} \mathbb{O}(3)$-модели. Например, лоренцева калибровка для $\mathbb{S}(3)$-связности приведет к уравнениям главного кирального поля для группы $\mathbb{S} \mathfrak{O}(3)$ в отсутствие дисклинаций. Таким образом, геометрическая теория дефектов при отсутствии дислокаций и дисклинаций сведется к уравнениям теории упругости для вектора смешений и к уравнениям главного кирального поля для спиновой структуры.

Благодарности. Автор выражает искреннюю благодарность И. В. Воловичу, Р. Дандолову, Е. А. Иванову и А.И. Пашневу за обсуждение статьи и полезные замечания. Работа выполнена при поддержке РФФИ, гранты № 96-15-96131 и 02-01-01084. Гостеприимство Института Эрвина Шредингера (Вена) принимается с большой благодарностью.

\section{Список литературы}

[1] T. H. R. Skyrme. Proc. Roy. Soc. London. A. 1961. V. 260. P. 127-138.

[2] В.Е. Захаров, С.В. Манаков, С. П. Новиков, Л. П. Питаевский. Теория солитонов: Метод обратной задачи. М.: Наука, 1980; Р. Раджараман. Солитоны и инстантоны в квантовой теории поля. М.: Мир, 1985; Л.А. Тахтаджян, Л.Д. Фаддеев. Гамильтонов подход в теории солитонов. М.: Наука, 1986; W. J. Zakrzewski. Low Dimensional Sigma Models. Bristol-Philadelphia: Adam Hilger, 1989; Ю. П. Ръъбаков, В. И. Санюк. Многомерные солитоны. М.: Изд-во РУДН, 2001.

[3] А. А. Белавин, А. М. Поляков. Письма в ЖЭТФ. 1975. Т. 22. № 10. С. 503-506.

[4] Дж. Эллиот, П. Добер. Симметрия в физике. Т. ІІ. М.: Мир, 1983.

[5] Б. А. Дубровин, С. П. Новиков, А. Т. Фоменко. Современная геометрия. Методы и приложения. Изд. 4. М.: Наука, 1998.

[6] Л. Д. Фаддеев. В поисках многомерных солитонов. В сб.: Нелокальные, нелинейные и неренормируемые теории поля. Дубна: ОИЯИ, 1976. С. 207-223.

[7] L. Faddeev, A. J. Niemi. Phys. Rev. Lett. 1999. V. 82. P. 1624-1627; Nature. 1997. V. 387. P. 58-66; R. A. Battye, P. Sutcliffe. Phys. Rev. Lett. 1998. V. 81. P. 4798-4801; Proc. Roy. Soc. London. A. 1999. V. 455. P. 4305-4331; J. Hietarinta, P. Salo. Phys. Lett. B. 1999. V. 451. P. $60-67$.

[8] M. O. Katanaev, I. V. Volovich. Ann. Phys. 1992. V. 216. P. 1-28; 1999. V. 271. P. 203-232.

[9] М. О. Катанаев. ТМФ. 2003. Т. 135. № 2. С. 338-352.

Поступила в редакцию 17.XII.2002 г., после доработки 14.IV.2003 г. 